\title{
La alergología en el sistema sanitario público de la Comunidad de Madrid: situación actual y oportunidades en el sistema sanitario público del siglo XXI
}

\section{Allergology in the public health service of the Community of Madrid: current situation and opportunities in the XXI century public health system}

\author{
J. Domínguez-Ortega ${ }^{1}$, J. Ruiz Hornillos ${ }^{2}$, T. Chivato ${ }^{3}$, B. De la $\mathrm{Hoz}^{4}$, A. Elices ${ }^{5}$, \\ A. Iglesias ${ }^{6}$, S. Quirce ${ }^{1}$, F. Vega ${ }^{7}$, M. Rodríguez Mosquera ${ }^{6}$
}

\section{RESUMEN}

La alta prevalencia de las enfermedades alérgicas y una mayor complejidad diagnóstica y terapéutica, exigen un alergólogo altamente capacitado profesionalmente y organizaciones sanitarias conocedoras de los recursos humanos y estructurales disponibles. En España faltan datos actualizados en alergología e indicadores de actividad que reflejen esta complejidad. Este trabajo analiza la situación, detecta necesidades y plantea soluciones de mejora para la alergología en la Comunidad de Madrid.

Los 24 jefes de servicio de Alergología de los hospitales públicos madrileños con asistencia alergológica, completaron voluntariamente la encuesta diseñada específicamente para este trabajo, con datos de actividad asistencial (2010), docencia e investigación (2009-2010). Se realizó un análisis DAFO y se diseñaron líneas estratégicas y propuestas de mejora orientadas a adoptar las soluciones más idóneas.

Un total de 117 alergólogos (41\% doctores) en el sistema público de salud $(4,6$ profesionales/centro; $\leq 3$ en 10 hospitales), realizaban consulta externa (4000 primeras consultas/ hospital/año, 36\% de alta resolución; 6200 visitas sucesivas/ hospital/año). Se ejecutaban procedimientos básicos diagnósticos y terapéuticos, incluyendo pruebas complejas propias de hospital de día, mayoritariamente no reconocidas en los indicadores de actividad. Dos hospitales tenían hospitalización propia y 10 disponían de acreditación docente (2,78 MIR/año). Bianualmente, un servicio participaba en 4 proyectos de investigación, 2 ensayos clínicos, 16 publicaciones y 17 comunicaciones a congresos, con notables diferencias entre hospitales.

Madrid cuenta con alergólogos bien preparados y una organización asistencial alergológica adecuada aunque posiblemente insuficiente. Nuevas herramientas de registro de actividad y el incremento de la cooperación corporativa, aprovechando nuevas tecnologías y la proximidad geográfica, mejorarían la calidad y la eficiencia.

Palabras clave. Alergia. Gestión clínica. Indicadores asistenciales. Madrid. Sanidad pública.

\section{ABSTRACT}

The high prevalence of allergic diseases and the use of more complex diagnostic techniques and therapeutic options require allergists to be highly trained professionals and healthcare organizations to be knowledgeable about available structural and human resources. Updated information is lacking in Spain, and current activity indicators do not reflect complexity The present article analyzes the situation of allergology in Madrid and proposes improvements where necessary.

The heads of the allergy departments of public hospitals in Madrid voluntarily completed a purpose-designed survey. Data on care activity (2010) and on teaching and research activity (2009-2010) were provided. A SWOT analysis was performed, and strategic lines and proposals for improvement were designed.

One hundred and seventeen allergists ( $41 \%$ with a PhD) worked in 24 hospitals with an allergology department (4.6 physicians/center; $\leq 3$ in 10 hospitals). All the institutions had an outpatient clinic (4,000 first consultations/hospital/year, $36 \%$ high-resolution rate; 6200 successive visits/hospital/year) performing complex diagnostic and therapeutic procedures most of which were not taken into account by activity indicators. Two hospitals had their own hospitalization facilities and 10 were accredited teaching hospitals (2.78 medical residents/ year). The survey revealed that, twice yearly, a typical allergology service participated in 4 research projects, 2 clinical trials, 16 publications and 17 communications at scientific meetings, with notable differences between hospitals.

Allergists in Madrid are well prepared. The allergology care structure is adequate, although possibly insufficient. Quality and efficiency can be improved with new tools for recording activity and by increasing coordination and taking advantage of new technologies and geographical proximity.

Key words. Allergy. Clinical management. Care indicators. Madrid. Public health.

\section{An. Sist. Sanit. Navar. 2014; 37 (3): 317-327}

1. Servicio de Alergia. Hospital Universitario La Paz. IDIPAZ. Madrid.

2. Unidad de Alergología. Hospital Infanta Elena. Valdemoro. Madrid.

3. Facultad de Medicina. Universidad CEU San Pablo. Madrid.

4. Servicio de Alergia. Hospital Universitario Ramón y Cajal. IRyCIS. Madrid.

5. Sección de Alergología. Hospital del Henares. Coslada. Madrid.

6. Servicio de Alergia. Hospital Universitario Puerta de Hierro. Majadahonda. Madrid.

7. Servicio de Alergia. Hospital Universitario de la Princesa. Madrid.

\section{Correspondencia:}

Dr. Javier Domínguez Ortega

Servicio de Alergia. IDIPAZ

Hospital Universitario La Paz

Paseo de la Castellana, 261

28046 Madrid

E-mail: javier.dominguez@idipaz.es

Recepción: 11 de febrero de 2014

Aceptación provisional: 3 de abril de 2014

Aceptación definitiva: 18 de junio de 2014 


\section{INTRODUCCIÓN}

Existen evidencias de que la prevalencia de las enfermedades alérgicas ha aumentado en los últimos años en todo el mundo ${ }^{1,2}$, especialmente en países industrializados, constituyendo hoy un problema global de salud. En España, la patología alérgica supone el 3-5\% de las consultas de Atención Primaria (AP) ${ }^{3}$. La rinitis alérgica afecta al $22 \%$ de la población ${ }^{4}$ y la prevalencia del asma acumulada se estima en el $6,2 \%$ en niños de $6-7$ años y $11 \%$ a los 13 $14^{5}$. En adultos, ronda el $5 \%{ }^{6}$. La alergia a alimentos afecta a casi el $5 \%$ de la población adulta, alcanzando el $8 \%$ en población pediátrica?

La Comunidad de Madrid (CM) no permanece al margen de ese incremento. La actividad especializada alergológica constituye el $7 \%$ de todas las derivaciones desde AP y el 5\% de todas las primeras visitas anuales realizadas. Alergología constituye la quinta especialidad más consultada ${ }^{8}$. Considerando que, al menos una cuarta parte de la población padece algún tipo de enfermedad alérgica, podemos inferir que en la $\mathrm{CM}$ existen aproximadamente 1,5 millones de enfermos alérgicos. Puesto que, además, la prevalencia tiende a aumentar, especialmente en población infantil, es previsible que, en los próximos años, sigan siendo una carga en cuanto al malestar y pérdida de calidad de vida para los individuos afectados, impliquen un alto coste social y económico para las familias y, más ampliamente, para las administraciones públicas, que constituyen las principales financiadoras del sistema sanitario español ${ }^{9}$. La alergia implica costes indirectos, como absentismo laboral o menor rendimiento profesional y escolar; costes intangibles como los derivados de la afectación de la calidad de vida, y, obviamente costes directos como el gasto sanitario en consultas médicas, ingresos hospitalarios o fármacos ${ }^{10}$. En este sentido, un diagnóstico alergológico correcto es fundamental. Por ejemplo, sólo el 6-10\% de las reacciones adversas con fármacos están asociadas a un mecanismo alérgico ${ }^{11}$ y la falta de diagnóstico específico puede multiplicar por 4 el coste de un tratamiento $^{12}$. Del mismo modo, un $20 \%$ de la población altera su dieta por haber presentado una reacción adversa a un alimento ${ }^{13}$, pero la alergia a los alimentos por mecanismo IgE-mediado, sólo afecta aproximadamente al $6 \%$ de los niños y al $3,7 \%$ de los adul$\operatorname{tos}^{14}$.

La alergología es la especialidad médica que comprende el conocimiento, diagnóstico y tratamiento de la patología producida como consecuencia de un mecanismo de hipersensibilidad, así como las técnicas que le son propias ${ }^{15}$. Se ha convertido en una especialidad multidisciplinar, que atiende a pacientes de cualquier edad, estudiando las consecuencias sistémicas del proceso alérgico y sus manifestaciones locales en un determinado órgano o sistema. A medida que se avanza en la comprensión de la fisiopatología, se acompaña de mayor complejidad diagnóstica. Además, nuevas líneas terapéuticas condicionan una modificación de la práctica clínica tradicional. La mejora en calidad de vida genera un incremento de la demanda asistencial especializada, entre otras cosas, porque los usuarios de la sanidad cada vez son más conscientes de las posibilidades de solución de sus problemas de salud. Por todo ello, y puesto que es el Sistema Nacional de Salud el que garantiza la adecuada atención alergológica en condiciones de equidad y eficiencia, las necesidades de formación, docencia y la propia organización de los recursos asistenciales disponibles, en su sentido más amplio, también han de actualizarse de acuerdo a esa nueva realidad, especialmente en contextos económicos adversos y ante enfermedades de curso crónico como son las alérgicas ${ }^{16}$.

Sin embargo, no existen datos publicados recientes de la organización sanitaria para la alergología en España y, particularmente, en la CM. Además, la codificación de enfermedades alérgicas basada en CIE-9 está escasamente distribuida ${ }^{17}$. En la clasificación CIE-10, las enfermedades alérgicas están dispersas en diferentes epígrafes ${ }^{18}$, por lo que resulta poco funcional en la práctica diaria. Tampoco se dispone 
de indicadores específicos y adecuados, medibles y evaluables con criterios iguales en todas las unidades de alergología. Los indicadores asistenciales actuales solo miden actividad de primeras consultas y sucesivas, sin ninguna relación con la complejidad de los procesos atendidos, de los métodos diagnósticos y de los procedimientos terapéuticos, que exigen profesionales preparados y condiciones adecuadas que garanticen la seguridad del paciente y la calidad del procedimiento.

Por ello, conscientes de esta necesidad de información, con el primer objetivo de hacer un análisis de la situación, detectar necesidades y plantear posibles soluciones de mejora, presentamos el trabajo realizado para establecer una aproximación detallada de esta realidad de la atención sanitaria en alergología en el sistema sanitario público de la CM.

\section{MÉTODOS}

Se solicitó la colaboración voluntaria de alergólogos que desarrollaran su actividad profesional en los servicios de alergología en cualquiera de los hospitales con actividad especializada del SERMAS (Servicio Madrileño de Salud) durante el primer semestre de 2011. Se dividieron en 5 grupos o comisiones: concepto de la especialidad de alergología; modelos de relación entre alergólogos; modelos de relación con otras especialidades; modelos de relación con AP y servicios de urgencias; futuro de la especialidad. Se integraban en ellas especialistas de hospitales de diferente grupo asistencial, disminuyendo así sesgos y consiguiendo una visión global de la situación y las propuestas de mejora. Se estableció un coordinador principal del trabajo y un secretario redactor que, junto a los coordinadores de las comisiones formaban un grupo técnico, encargado del análisis final y la redacción homogénea de las propuestas de mejora. Se eligió un grupo asesor, integrado por profesionales de reconocido prestigio de la alergología madrileña, coordinado a su vez por la presidenta de la Sociedad de Madrid Castilla-La Mancha de Alergología e Inmunología Clínica, que fueron consultados para aspectos concretos del trabajo.

En una primera fase, con la finalidad de llevar a cabo el análisis descriptivo de la actividad, se diseñó una encuesta específica y exclusiva para este estudio, que fue remitida a los jefes de servicio y responsables de unidad que completaron voluntariamente. La encuesta recogió datos de actividad asistencial, docencia e investigación y los datos se referenciaron al cierre del año 2010 para actividad asistencial y el bienio 2009-2010 para docencia e investigación.

Al no disponer de un modelo de referencia o un patrón oro con el que comparar la actividad de alergología, se confeccionó un estándar de actividad en técnicas y procedimientos terapéuticos mediante el cálculo de los parámetros incluidos en la cartera de servicios propuesta por la Sociedad Española de Alergología e Inmunología Clínica (SEAIC) ${ }^{19}$ y el documento de posición de la World Allergy Organization $(\mathrm{WAO})^{20}$. Se dividieron en dos grupos según el grado de complejidad (Tabla 1), unas de menor riesgo y complejidad (grupo A) y otras que requieren la infraestructura de hospital de día (grupo B) por las propias características de la prueba o por el riesgo de desarrollar una reacción alérgica durante la realización de la misma ${ }^{21-23}$. Se valoró previamente en una muestra de 4 hospitales representativos. Las encuestas se recogieron en formato Excel (2003) y tras su recepción, se procedió a su análisis estadístico descriptivo, estableciendo las medias y medianas para las variables cuantitativas y describiéndose con el porcentaje de distribución para las cualitativas. En los casos en que no había información correspondiente a una determinada prueba, se consideró no realizada en dicho hospital y se valoró como 0 . Si constaba como realizada, pero sin aporte de datos, el hospital en cuestión se excluía del cálculo estadístico, aunque sí se constataba que ejecutaba la prueba. 
Tabla 1. Clasificación de las pruebas complementarias realizadas en Alergología

\begin{tabular}{ll}
\hline \multicolumn{1}{c}{ Grupo A } & \multicolumn{1}{c}{ Grupo B } \\
\hline Pruebas cutáneas & Provocación bronquial inespecífica (metacolina, manitol, adenosina) \\
\hline Pruebas epicutáneas & Provocación bronquial específica (sustancias de alto y bajo PM) \\
\hline Espirometría & Provocación con alimentos \\
\hline Test de broncodilatación & Provocación con aditivos \\
\hline Esputo inducido & Provocación con medicamentos \\
\hline Oscilometría de impulsos & Desensibilización a medicamentos \\
\hline Rinomanometría & Desensibilización a alimentos \\
\hline Endoscopia nasal & Administración de fármacos biológicos \\
\hline Citología nasal & Inmunoterapia de riesgo \\
\hline Olfatometría & Provocación nasal específica \\
\hline
\end{tabular}

Grupo A. Pruebas realizadas en los hospitales consideradas de complejidad baja en cuanto a consumo de tiempo facultativo y recursos del servicio destinados a su realización y/o riesgo bajo de reacción.

Grupo B. Pruebas realizadas en ambiente hospitalario de complejidad alta en cuanto a consumo de tiempo de facultativo y recursos del servicio destinados a su realización y/o mayor riesgo de reacción.

Finalmente, se realizó un análisis DAFO de la situación de partida, para la detección de problemas e identificación de necesidades, pero también de las fortalezas como especialidad en el SERMAS (Tabla 2). Posteriormente, cada comisión diseñó las líneas estratégicas específicas en su materia de trabajo, estableciendo propuestas de mejora concretas basadas en medidas de actuación definidas y orientadas a adoptar las soluciones más idóneas.

Tabla 2. Principales conclusiones del análisis DAFO

\section{Fortalezas}

- Proximidad entre centros hospitalarios y especialistas.

- Buena formación especializada de los alergólogos madrileños.

- Amplia tradición docente e investigadora.

\section{Debilidades}

- Desconocimiento de la especialidad entre gestores y otros especialistas

- Ausencia del reconocimiento de la complejidad de los métodos diagnósticos y terapéuticos específicos de la especialidad.

- Falta de la materia Alergología en los planes de pre-grado de la mayoría de las facultades de Medicina.

\section{Amenazas}

- Aumento de la prevalencia de enfermedades alérgicas sin una correcta adecuación de los recursos.

\section{Oportunidades de mejora}

- Facilitar las redes de cooperación corporativa (asistencial, docente y de investigación) aprovechando la proximidad geográfica y personal, así como las nuevas tecnologías.

- Establecer indicadores de actividad asistencial que reflejen la complejidad de la atención especializada alergológica, permitiendo con ello la indicación de tiempos asistenciales y los medios personales y materiales por procesos que garanticen la calidad asistencial y la seguridad del paciente.

- Potenciar el acceso de los médicos alergólogos a los departamentos de medicina en las universidades madrileñas. 


\section{RESULTADOS}

Participaron 55 profesionales, 51 alergólogos y 4 médicos de AP integrados en esa comisión específica. Se obtuvo respuesta de los 24 hospitales madrileños con actividad especializada de alergología en 2011. La última encuesta cumplimentada se recibió en diciembre de 2011.

Entre estos 24 hospitales, 9 pertenecían al nivel III, 9 al nivel II y 4 al nivel I. Se incluyeron 2 hospitales de apoyo a la asistencia sin área de referencia propia. En 16 centros (67\%) se atendía a pacientes alérgicos de cualquier edad, 7 sólo a mayores de 14 años y en 1, exclusivamente a la población pediátrica. Trece hospitales $(54,2 \%)$ contaban con jefatura de servicio propia, siendo en los 11 restantes una sección o unidad del área médica, dependiente de Medicina Interna o directamente de Dirección Médica. Se contabilizaron 117 médicos alergólogos, con distribución desigual en los diferentes hospitales, siendo la media de 4,6 profesionales, con un mínimo de uno y un máximo de 12 en un solo centro. Diez hospitales contaban con una plantilla $\leq 3$ especialistas. El ratio enfermería/médico fue de 0,93 .
Solo 2 hospitales contaban con camas propias, aunque en la mayoría ingresaban pacientes a cargo de alergología en camas de los servicios de Medicina Interna o UCI. Todos los servicios realizaban una notable actividad asistencial en consulta externa. El número total de primeras visitas en alergología durante 2010 fue de 96.775 en toda la CM. En promedio, sin valorar complejidad asistencial ni ajuste por plantilla, un servicio de Alergología realizaría 4.000 primeras consultas/año, lo que implicaría cerca de 1.000 primeras visitas anuales por médico especialista. El 36\% de ellas serían de alta resolución, realizándose pruebas complementarias en el mismo día y permitiendo el diagnóstico y una orientación terapéutica. Además, se realizaron más de 141.000 visitas sucesivas con un promedio de 6.200 por hospital (índice medio de sucesivas/primeras 1,58) observándose, no obstante, grandes diferencias entre hospitales. Estos registros se superaban en 13 de los 24 hospitales (Fig. 1). De media, el 84\% de las primeras consultas se originaron desde AP. Además, cada servicio atendía 17-18 interconsultas de pacientes ingresados por mes. La demora media para primera consulta se estableció en 30 días (2-90).

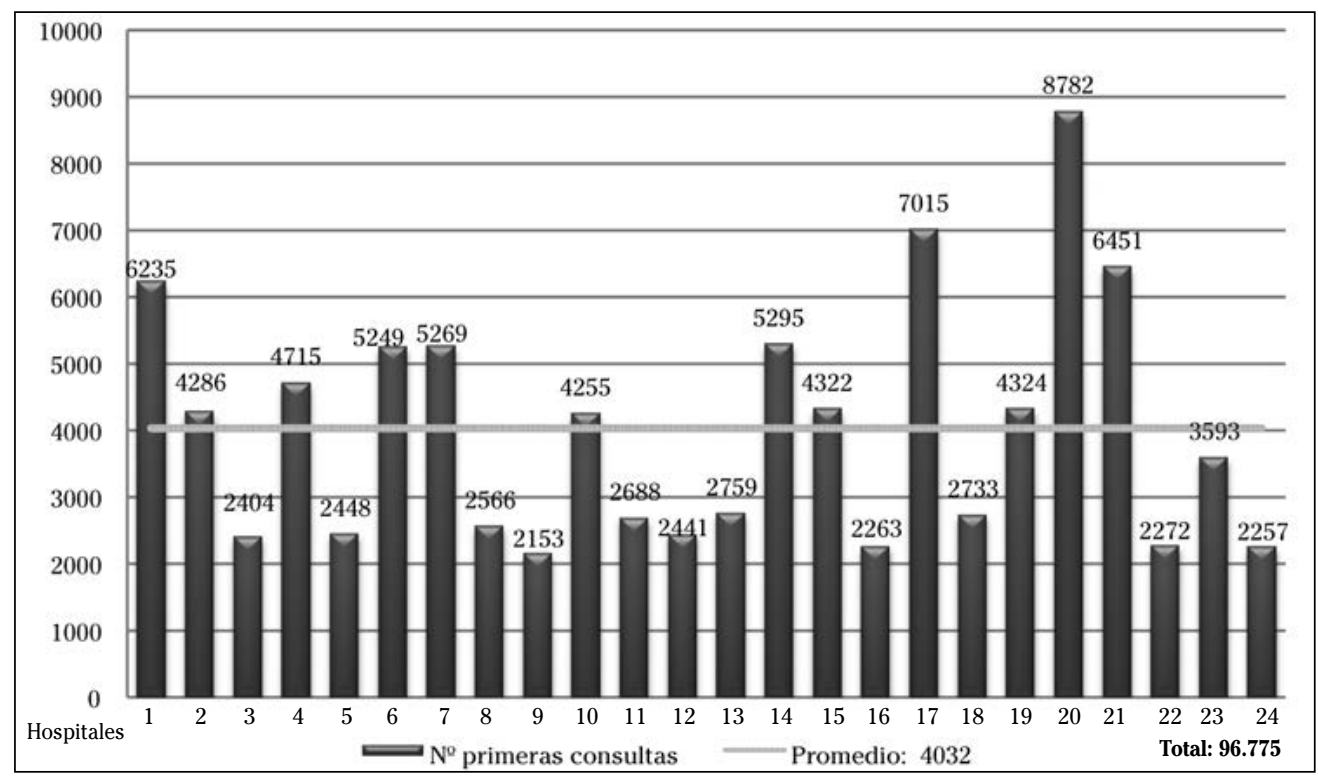

Figura 1. Número de primeras consultas en cada uno de los hospitales de la CM. 
La encuesta reflejó la existencia de dos perfiles de servicios de alergología en relación con los propios hospitales donde se encontraban. La actividad vendría marcada por la propia evolución de los hospitales y la población atendida. Unos, en grandes hospitales, muy consolidados, con desarrollo de líneas de investigación y seguimiento de patologías específicas, con población mayoritaria de enfermos de revisión. Otros, de reciente creación, en hospitales nuevos, con amplia población demandante de atención alergológica por primera vez. En ambos tipos, es decir, en los 24 hospitales, se ejecutaban los procedimientos básicos de diagnóstico y tratamiento, incluyendo pruebas complejas como exposición a alimentos o medicamentos, propias de actividad de hospital de día (Tabla 3). Sin embargo, mayoritariamente esta actividad de hospital de día no estaba reconocida específicamente en los indicadores de actividad. Los valores absolutos de las pruebas y procedimientos terapéuticos realizados, se describen en la tabla 4.

Tabla 3. Número de servicios de alergología que realizan cada exploración complementaria del grupo B o de mayor complejidad y riesgo

\begin{tabular}{lc}
\hline \multicolumn{1}{c}{ Grupo B } & $\begin{array}{c}\text { No servicios de alergia que } \\
\text { realizan cada exploración } \\
\text { complementaria* }\end{array}$ \\
\hline Provocación bronquial inespecífica (metacolina, manitol, adenosina) & 8 \\
\hline Provocación bronquial específica (sustancias de alto o bajo PM) & 9 \\
\hline Provocación nasal específica & 11 \\
\hline Provocación conjuntival específica & 17 \\
\hline Provocación o test de tolerancia con alimentos/aditivos & 23 \\
\hline Provocación o test de tolerancia con medicamentos & 24 \\
\hline Desensibilización a medicamentos o quimioterápicos & 15 \\
\hline Desensibilización a alimentos & 9 \\
\hline Administración de tratamientos biológicos (Omalizumab) & 20 \\
\hline Inmunoterapia de riesgo & 19 \\
\hline
\end{tabular}

* De un total de 24.

Con respecto a los aspectos docentes y de investigación, el $41 \%$ de los alergólogos del SERMAS, habían obtenido el título de doctor; 10/24 de los hospitales disponían de acreditación docente para formación MIR y 2 más estaban en proceso de acreditación, con una media de 2,78 plazas MIR por hospital acreditado. Se observó una carencia de formación de pregrado de alergología con sólo 5 profesores asociados en cualquiera de las universidades madrileñas. Sin embargo, la actividad investigadora estaba presente en la mayoría de los servicios, y el $62,5 \%$ había participado al menos en un ensayo clínico en los 2 últimos años, 19/24 en proyectos de investigación y el $67 \%$ habían presentado comunicaciones en congresos internacionales. En promedio bianual (2009-2010) un servicio de alergología estaría implicado en 4 proyectos de investigación, 2 ensayos clínicos, 16 publicaciones, 4 ponencias y 17 comunicaciones a congresos, si bien existían notables diferencias entre los hospitales, no necesariamente por la categoría asistencial de los mismos. 
Tabla 4. Pruebas diagnósticas complejas y procedimientos terapéuticos realizados en el servicio de Alergología en el año 2010

\begin{tabular}{|c|c|c|c|c|c|c|c|}
\hline Pruebas & $\mathbf{X}$ & SD & Mediana & Mínimo & Máximo & Quartil 25 & Quartil 75 \\
\hline Pruebas cutáneas & 48066,9 & 101836 & 4300 & 1433 & 426736 & 2927 & 33504,5 \\
\hline Pruebas epicutáneas & 2196,61 & 6056,31 & 462 & 89 & 29202 & 304 & 980 \\
\hline Provocación con sulfitos & 5,08 & 9,82 & 0 & 0 & 40 & 0 & 5,25 \\
\hline Provocación conjuntival & 17,96 & 25,18 & 1,5 & 0 & 98 & 0 & 30,5 \\
\hline Provocación nasal & 3,71 & 7,75 & 0 & 0 & 30 & 0 & 2,75 \\
\hline $\begin{array}{l}\text { Prov. bronquial inespecífica } \\
\text { con metacolina }\end{array}$ & 105,35 & 132,54 & 31 & 0 & 417 & 0 & 192 \\
\hline $\begin{array}{l}\text { Provocación bronquial con } \\
\text { manitol }\end{array}$ & 4,92 & 10,97 & 0 & 0 & 50 & 0 & 4,25 \\
\hline $\begin{array}{l}\text { Provocación bronquial con } \\
\text { adenosina }\end{array}$ & 3,37 & 11,43 & 0 & 0 & 41 & 0 & 0 \\
\hline $\begin{array}{l}\text { Provocación bronquial } \\
\text { específica con sustancias de } \\
\text { alto peso molecular }\end{array}$ & 7,25 & 16,43 & 0 & 0 & 57 & 0 & 2,25 \\
\hline $\begin{array}{l}\text { Provocación bronquial con } \\
\text { sustancias de bajo peso } \\
\text { molecular }\end{array}$ & 4,29 & 13,75 & 0 & 0 & 62 & 0 & 0 \\
\hline $\begin{array}{l}\text { Desensibilización con } \\
\text { alimentos }\end{array}$ & 56,35 & 168,41 & 0 & 0 & 628 & 0 & 12,5 \\
\hline $\begin{array}{l}\text { Desensibilización con } \\
\text { fármacos }\end{array}$ & 6,96 & 12,76 & 3,5 & 0 & 60 & 0 & 8,5 \\
\hline $\begin{array}{l}\text { Desensibilización con } \\
\text { quimioterápicos }\end{array}$ & 22,17 & 52,58 & 2 & 0 & 228 & 0 & 10,5 \\
\hline $\begin{array}{l}\text { Administración de fármacos } \\
\text { biológicos }\end{array}$ & 131,33 & 226,58 & 12 & 0 & 1000 & 5 & 185 \\
\hline Inmunoterapia inhalantes & 1454,3 & 2664,81 & 738 & 0 & 12633 & 31 & 1728,5 \\
\hline Inmunoterapia himenópteros & 212,95 & 472,24 & 25 & 0 & 2000 & 6,25 & 185 \\
\hline
\end{tabular}

\section{DISCUSIÓN}

El médico alergólogo es el único con la titulación necesaria según la legislación española para atender pacientes alérgicos de cualquier edad ${ }^{15}$. Apoyado en su acción por los profesionales de enfermería entrenados para ello, lleva a cabo los procedimientos diagnósticos y terapéuticos específicos, algunos de gran complejidad. Disponer de datos actualizados y fiables permite la planificación de soluciones efectivas y eficientes, facilitando además la adaptación de esos recursos a las previsibles demandas futuras. La realidad es que en España no se dispone hoy de esos datos ${ }^{24,25}$.

Utilizando la encuesta como fuente de información, se ha presentado una visión actualizada de los recursos humanos y materiales disponibles para satisfacer la demanda sanitaria alergológica en el sistema sanitario público de la CM. Constituye la primera experiencia en España en este sentido, al menos tan en profundidad. Desde el punto de vista científico, la metodología de recogida de datos mediante una encuesta podría tener sesgos de correspondencia con la realidad, ya que no se 
realizó ninguna comprobación por otros medios de los datos aportados. Sin embargo, creemos que resulta útil para la recopilación de datos cuantitativos al reflejar transversalmente aspectos específicos de la realidad cotidiana de los diferentes servicios de alergología. De esta forma, el modelo ofrecía unas claras ventajas sobre las encuestas oficiales a las que hemos tenido acceso $^{8}$. Éstas, solo reflejan la parte registrada mediante los sistemas de información actuales, desarrollados especialmente para patología hospitalaria, y no tanto para medir la actividad alergológica, mayoritariamente ambulatoria y muy variable en complejidad diagnóstica y terapéutica. De hecho, esta metodología ha sido la base de lo que constituye el plan estratégico de la especialidad en la $\mathrm{CM}^{26}$.

El trabajo resulta representativo al contar con la respuesta de todos los responsables de los diferentes servicios y unidades especializadas. Además el número de especialistas participantes en el trabajo alcanzó el $41,9 \%$ de todos los que trabajaban en el SERMAS en ese momento. Este hecho corrobora los datos publicados previamente, que constataban que el alergólogo español es, en general, un profesional implicado con sus pacientes y comprometido con la especialidad $^{27}$, que asume una importante carga asistencial como se deriva de los datos de la encuesta, sin olvidar otros aspectos relevantes en la vida profesional de un médico, como la docencia y la investigación como también refleja este trabajo. Sin embargo, es destacable que la alergología, a pesar de la alta prevalencia de las enfermedades alérgicas, no está incluida en la formación universitaria en las universidades públicas madrileñas. Sin embargo, la SEAIC, en su nuevo plan estratégico apuesta por modificar esta situación ${ }^{19}$. Sin duda, un mejor conocimiento de la patología por los estudiantes de medicina, redundaría en el futuro en mejores derivaciones a atención especializada y una gestión más eficiente de los recursos disponibles.

$\mathrm{Al}$ analizar los datos, se observa una amplia heterogeneidad en las definiciones utilizadas en los diferentes registros. Es especialmente significativo en el concep- to de alta resolución o en los registros de pruebas cutáneas y epicutáneas, ya que algunos hospitales registran por paciente y otros por cada uno de los alérgenos testados (cada batería de pruebas suele incluir alrededor de 20 alérgenos de media). Por ello, es indispensable contar con una definición de cartera de servicios actualizada y una normalización del registro pormenorizado de la actividad asistencial, teniendo en cuenta no solo la actividad de consulta y en base a criterios de calidad y eficiencia. Para ello, se debería tener en cuenta la valoración por grupo asistencial del hospital al que se pertenece, la complejidad de la patología atendida y la población derivada desde otras áreas hospitalarias y contabilizar los tiempos empleados por el especialista en la realización y supervisión de pruebas diagnósticas específicas y procedimientos terapéuticos.

En este sentido, aunque todos los servicios de alergología reconocen que realizan actividades en las que sería necesario contar con un hospital de día, son muy pocos los que la tienen reconocida en la estadística oficial de la CM. Sería necesario armonizar esta actividad en los distintos servicios de alergología, dotándolos de un área específica en la que realizarla, con el equipamiento y los recursos humanos necesarios, ofreciendo al paciente toda la seguridad requerida, estableciendo estándares de calidad asistencial, como la asignación de un facultativo exclusivamente a la supervisión de dichas actividades.

La combinación de conocimiento científico y la proximidad geográfica en la CM, ofrece multitud de oportunidades para el desarrollo de cooperación corporativa, facilitando la generación de redes de expertos que impulsen y compartan ese conocimiento. En este punto se han propuesto varias líneas de actuación como la creación de un entorno-web corporativo para todos los alergólogos del SERMAS, que permitiera promover la formación y la adquisición de capacidades para la investigación, así como el intercambio de información y mejora de la comunicación entre profesionales. Otras líneas de actuación serían la estandarización del lenguaje empleado para el registro 
de actividad, con la creación de indicadores de asistencia, calidad y científico-técnicos adecuados, o la identificación de necesidades formativas para satisfacerlas en coordinación con las sociedades científicas. Además, esa cercanía física abre la puerta a potenciar vías de derivación de pacientes, e incluso, exclusivamente, las muestras para diagnóstico, mejorando la eficiencia. En este sentido, aplicando criterios de eficiencia, las exploraciones más sofisticadas o complejas, se podrían agrupar en hospitales de referencia, garantizándose que esta disponibilidad fuera conocida por los especialistas y fácilmente accesible.

En general, la distribución de servicios asistenciales de alergología en la CM es mejor que en otras regiones españolas, próximo a un alergólogo por cada 50.000 habitantes que recomienda la OMS, si bien en el mundo la razón alergólogo/habitantes es muy variable, oscilando incluso entre los 1:25 millones de algunos países africanos a 1:16.000 habitantes en Alemania ${ }^{28}$. En España, según datos del Ministerio de Sanidad de $2009^{29}$, solo La Rioja o Castilla-La Mancha alcanzarían el número recomendado mientras que en otras comunidades españolas como Navarra, Canarias o Extremadura se aproximaría al mismo y, sin embargo en algunas, se observan claras deficiencias. En Baleares no existen especialistas de alergología en el sistema sanitario público, y en Cataluña el número de alergólogos es claramente inferior a Madrid a pesar de tener poblaciones similares ${ }^{29}$.

En resumen, los resultados de este trabajo reflejan la atención alergológica en el SERMAS, que cuenta con una red de profesionales bien preparados y una organización asistencial adecuada, pero posiblemente insuficiente si se contempla la gran complejidad de los nuevos procedimientos diagnósticos y terapéuticos en Alergología. Además, se deberían crear nuevas herramientas de registro de actividad recogiendo esa complejidad, e implementar medidas de cooperación corporativa entre profesionales y servicios, que permitan una atención sanitaria más eficiente, aprovechando las nuevas tecnologías y la proximidad geográfica.
Agradecimientos

Agradecimientos a los 52 médicos participantes en el estudio.

\section{BIBLIOGRAFÍA}

1. Izquierdo-Domínguez A, Valero AL, Mullol J. Comparative analysis of allergic rhinitis in children and adults Curr Allergy Asthma Rep 2013; 13: 142-151.

2. Bousquet J, Schünemann HJ, Samolinski B, Demoly P, BaEna-CAGnani CE, Bachert $\mathrm{C}$ et al. Allergic rhinitis and its impact on asthma (ARIA): achievements in 10 years and future needs. J Allergy Clin Immunol 2012; 130: 1049-1062.

3. Castillo JA, Navarro A, Quirce S, Molina J, GarCíA-MArcos L, Julí B et al. Prevalence and characteristics of rhinitis in adult asthmatic patients attending allergist, primary care and pulmonologist settings in Spain (AIR study). Med Clin (Barc) 2011; 136: 284-289.

4. Navarro A, Colas C, Anton E, Conde J, Dávila I, Dordal MT et al. Rhinoconjunctivitis Committee of the SEAIC. Epidemiology of allergic rhinitis in allergy consultations in Spain: Alergologica-2005. J Investig Allergol Clin Immunol 2009; 19 (Suppl. 2): 7s-13s.

5. Arnedo-Pena A, García-Marcos L, Bercedo-Sanz A, Aguinaga-Ontoso I, González-Díaz C, GarcíaMerino A et al. Prevalence of asthma symptoms in schoolchildren and climate in Western European countries: an ecological study. Int J Biometeorol 2013; 57: 775-784.

6. Quirce S. Asthma in Alergologica 2005. J Investig Allergol Clin Immunol 2009; 19 (Suppl 2): 14-20.

7. Sicherer SH, SAMPSON HA. Food allergy: Epidemiology, pathogenesis, diagnosis, and treatment. J Allergy Clin Immunol 2014; 133 : 291-307.e5.

8. Dirección General de Hospitales. Consejería de Sanidad de la Comunidad de Madrid. 2010. http://www.madrid.org/cs/Satellite?ci $\mathrm{d}=1142344883673 \&$ language $=$ es \&pagename =PortalSalud $\% 2$ FPage $\% 2$ FPTSA_pintarConte nidoFinal\&vest=1142344883673. Acceso 16/ junio/2014

9. López-Casasnovas G, Costa-Font J, Planas I. Diversity and regional inequalities in the Spanish "system of health care services". Health Econ 2005; 14 (Suppl. 1): 221s-235s.

10. OJEDA P, SAnz DE BuRgoA V. Coste asma study. Costs associated with workdays lost and utilization of health care resources because of asthma in daily clinical practice in Spain. J Investig Allergol Clin Immunol 2013; 23: 234-241. 
11. Demoly P, Hillaire-Buys D. Classification and epidemiology of hypersensitivity drug reactions. Immunol Allergy Clin North Am 2004; 24: 345-356.

12. Sastre J, Manso L, Sánchez-García S, FernándezNiETo M. Medical and economic impact of misdiagnosis of drug hypersensitivity in hospitalized patients. J Allergy Clin Immunol 2012; 129: 566-567.

13. Sicherer SH, SAmpson HA. Food Allergy. J Allergy Clin Immunol 2006; 117: S470-S475.

14. Fernandez Rivas M. Food allergy in Alergologica 2005. J Investig Allergol Clin Immunol 2009; 19 (Suppl. 2): 37s-44s.

15. Real Decreto 127/1984, de 11 de enero, por el que se regula la formación médica especializada y la obtención del título de Médico Especialista. BOE número 26, de 31 de enero de 1984; 2524-2528.

16. García-Goñi M, Hernández-Quevedo C. Nuño-SoLINIS R, PAOLUCCI F. Pathways towards chronic care-focused healthcare systems: evidence of Spain. Health Policy 2012; 108: 236-245.

17. Simpson CR, Anandan C, Fischbacher C, Lefevre $\mathrm{K}$, SHEIKH A. Will systematized nomenclature of medicine-clinical terms improve our understanding of the disease burden posed by allergic disorders? Clin Exp Allergy 2007; 37: 1586-1593.

18. Preckova P, Zvarova J, Zvara K. Measuring diversity in medical reports based on categorized attributes and international classification systems. BMC Med Inform Decis Mak 2012; $12: 31$.

19. Sociedad Española de Alergología e Inmunología Clínica. www.seaic.org (último acceso 17/06/2014).

20. Del Giacco S, Rosenwasser LJ, Crisci CD, Frew AJ, KaLiner MA, LeE BW, et al. What is an allergist? Position statement of the WAO Specialty and Training Council. WAO Journal 2008; 1:19-20.

21. Diamant Z, Gauvreau GM, Cockcroft DW, Boulet LP, STERK PJ, DE JONGH FH et al. Inhaled allergen bronchoprovocation tests. J Allergy Clin Immunol 2013; 132: 1045-1055.
22. Calvani M, Berti I, Fiocchi A, Galli E, Giorgio V, MARTELl A et al. Oral food challenge: safety, adherence to guidelines and predictive value of skin prick testing. Pediatr Allergy Immunol 2012; 23: 755-761.

23. Demoly P, Adkinson NF, Brockow K, Castells M, Chiriac AM, Greenberger PA et al. International Consensus on drug allergy. Allergy 2014; 69 : 420-437.

24. García Pérez MA, Amaya Pombo C, Negro Álvarez JM. La alergología en España: pasado, presente y previsiones de futuro. Alergología Inmunología Clínica 2005; 20: 41-47.

25. Nieto A, Mazón A, Martín-Mateos MA, Plaza AM, Garde J, Alonso E et al. Pediatric allergy and immunology in Spain. Pediatr Allergy Immunol 2011; 22: 742-750.

26. Plan Estratégico de la Comunidad de Madrid para la especialidad de Alergología. Consejería de Sanidad. Dirección General de Hospitales. www.madrid.org

27. Chivato Pérez T, Campos Andreu A, Negro Álvarez JM, Caballero-Martínez F. Professional burnout and work satisfaction in Spanish allergists: analysis of working conditions in the specialty. J Investig Allergol Clin Immunol 2011; 21: 13-21.

28. Warner JO, Kaliner MA, Crisci CD, Del Giacco S, Frew AJ, Liu GH et AL. Allergy Practice Worldwide: A Report by the World Allergy Organization Specialty and Training Council. Int Arch Allergy Immunol 2006; 139: 166-174.

29. Barber Pérez P, González López-Valcárcel B, Suárez Vega R. Equipo de economía de la Salud de la Universidad de las Palmas de Gran Canaria. Oferta y necesidad de especialistas médicos en España 2010-2025. Ministerio de Sanidad, Política Social e Igualdad. Diciembre de 2011. Último acceso 17/06/2014. http://www.msssi.gob.es/profesionales/ formacion/necesidadEspecialistas/doc/11NecesidadesMEspecialistas(2010-2025).pdf 


\section{ADDENDUM \\ Participantes en el trabajo}

\section{COMITÉ ASESOR}

Coordinadora: Dra. Matilde Rodríguez Mosquera. Hospital Universitario Puerta de Hierro.

- Dr. Emilio Álvarez Cuesta. Hospital Universitario Ramón y Cajal.

- Dr. Ángel García Cubero. Clínica Alergasa.

- Dra. Consuelo Martínez-Cócera. Hospital Clínico Universitario San Carlos.

- Dra. María Rubio Sotes. Hospital Universitario Gregorio Marañón.

- Dr. Joaquín Sastre Domínguez. Fundación Jiménez Díaz.

- Dr. Ramón Vives Conesa. Hospital Universitario 12 de Octubre.

\section{COMITÉ TÉCNICO}

Comisión de trabajo

"Concepto de la Especialidad"

Coordinadora: Dra. Belén de la Hoz Caballer. Hospital Universitario Ramón y Cajal

- Dra. María Dolores Alonso Díaz de Durana. Fundación Hospital Alcorcón

- Dra. María Teresa Caballero Molina. Hospital Universitario La Paz.

- Dra. Inmaculada Cerecedo Carballo. Hospital del Sureste.

- Dra. Angélica Feliú Vila. Hospital del Tajo.

- Dra. Montserrat Fernández Rivas. Hospital Clínico San Carlos.

- Dra. María Isabel Garcimartín Galicia. Hospital Infanta Leonor.

- Dr. Pablo Rodríguez del Río. Hospital Infantil Universitario Niño Jesús.

- Dra. Ana María Umpiérrez Rodríguez. Hospital Carlos III.

- Dr. Francisco Vega de la Osada. Hospital de la Princesa.

\section{Comisión de trabajo}

"Modelos de relación con Atención Primaria y

Servicios de Urgencias"

Coordinadora: Dra. Ana Elices Apellániz. Hospital del Henares. Coslada

- Dr. Javier Contreras Porta. Hospital Universitario La Paz.

- Dra. Consolación de Frutos Moreno. Hospital de la Princesa.

- Dra. Marta Gema López San Martín. Hospital Universitario Puerta de Hierro.

- Dra. Ruth Mielgo Ballesteros. Hospital Universitario 12 de Octubre.

- Dra. Marta Reche Frutos. Hospital Infanta Sofía.

- Dra. Beatriz Rodríguez Jiménez. Hospital Universitario de Getafe.

- Dr. Javier Sola Martínez. Hospital Universitario Ramón y Cajal.

\section{CONSEJO ASESOR DE ATENCIÓN PRIMARIA}

- Dr. Eduardo Calvo Corbella. Centro de Salud Pozuelo Estación. SEMFYC.

- Dra. Carmen Valdés y Llorca. SEMERGEN.

- Dra. Agustina Cano Espín. SEMERGEN.

- Dr. Francisco José Sáez Martínez. SEMG
Comisión de trabajo

"Modelos de relación entre alergólogos"

Coordinador: Dr. Alfredo Iglesias Cadarso. Hospital Universitario Puerta de Hierro.

- Dr. Alberto Álvarez Perea. Hospital Universitario Gregorio Marañón.

- Dra. María del Carmen Diéguez Pastor. Hospital del Sureste

- Dra. Mar Gandolfo Cano. Hospital de Fuenlabrada.

- Dr. José Julio Laguna Martínez. Hospital de la Cruz Roja.

- Dra. Paloma Las Heras Almazán. Hospital de la Princesa.

- Dr. Francisco Javier Ruano Pérez. Hospital Infanta Leonor

- Dr. Javier Seoane Lestón. Hospital Severo Ochoa.

\section{Comisión de trabajo}

"Modelos de relación con otras especialidades"

Coordinador: Dr. Santiago Quirce Gancedo. Hospital Universitario La Paz

- Dra. María Luisa Baeza Ochoa de Ocariz. Hospital Universitario Gregorio Marañón.

- Dra. Ana María Fiandor Román. Hospital Universitario La Paz.

- Dra. Paloma Ibáñez Sandín. Hospital Infantil Universitario Niño Jesús.

- Dra. Mar Reaño Martos. Hospital Universitario Puerta de Hierro.

- Dra. Mercedes Rodríguez Rodríguez. Hospital Príncipe de Asturias.

- Dr. Miguel Ángel Tejedor Alonso. Fundación Hospital Alcorcón.

- Dra. Pilar Tornero Molina. Hospital Universitario Gregorio Marañón.

\section{Comisión de trabajo}

"Futuro de la especialidad"

Coordinador: Dr. Tomás Chivato Pérez. Hospital Central de la Defensa Gómez Ulla.

- Dra. Mar Fernández Nieto. Fundación Jiménez Díaz.

- Dr. David González de Olano. Hospital Universitario de Fuenlabrada.

- Dra. Aurora Losada Peña. Hospital Infanta Cristina.

- Dra. María Concepción López Serrano. Hospital Universitario La Paz.

- Dra. Tania Ramos. Hospital de la Princesa.

- Dra. Julia Rodríguez Rodríguez. Hospital Universitario 12 de Octubre.

- Dra. Silvia Sánchez García. Hospital Infantil Universitario Niño Jesús.

Redactor: Dr. Javier Ruiz Hornillos. Hospital Universitario Infanta Elena. Valdemoro

Coordinador general: Dr. Javier Domínguez Ortega. Hospital Universitario La Paz. 